\title{
Protein-Protein Interactions Induce pH-Dependent and Zeaxanthin-Independent Photoprotection in the Plant Light-Harvesting Complex, LHCII
}

Minjung Son ${ }^{\dagger}, \|$, Raymundo Moya ${ }^{\dagger}$, Alberta Pinnola ${ }^{\ddagger}$, Roberto Bassi ${ }^{\AA I}, \S$, and Gabriela S. Schlau-Cohen ${ }^{*, \dagger}$

${ }^{\dagger}$ Department of Chemistry, Massachusetts Institute of Technology, Cambridge, MA 02139, United States

"Iresent Address: Department of Chemistry, University of Wisconsin-Madison, Madison, WI 53706, United States

${ }^{\star}$ Department of Biology and Biotechnology, University of Pavia, 27100 Pavia, Italy

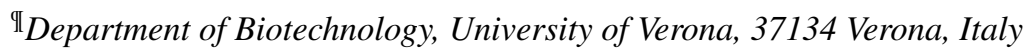

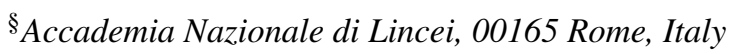




\section{EXPERIMENTAL SECTION}

\subsection{Sample preparation}

\subsubsection{Preparation of LHCII}

Thylakoid isolation. Stacked thylakoids were purified from leaves of spinach plants following a previously reported protoco ${ }^{1}$ with minor modifications. Tissues were harvested and freshly homogenized in cold extraction buffer $(0.5 \%$ milk powder, $400 \mathrm{mM} \mathrm{NaCl}, 20 \mathrm{mM}$ Tricine/KOH, $\mathrm{pH}$ 7.8, $2 \mathrm{mM} \mathrm{MgCl} 2$ and $5 \mathrm{mM}$ $\varepsilon$-aminocaproic acid, $1 \mathrm{mM}$ phenylmethylsulfonyl fluoride and $1 \mathrm{mM}$ benzamidine as protease inhibitors). After filtration, samples were precipitated by centrifugation at $4,000 \times \mathrm{g}$ for $10 \mathrm{~min}$ at $4{ }^{\circ} \mathrm{C}$ and then resuspended in hypotonic buffer $(15 \mathrm{mM} \mathrm{NaCl}, 5 \mathrm{mM} \mathrm{MgCl} 2,10 \mathrm{mM} \mathrm{HEPES} / \mathrm{KOH}, \mathrm{pH} 7.5$, and protease inhibitor). After centrifugation for $10 \mathrm{~min}$ at $10,000 \times \mathrm{g}$ at $4^{\circ} \mathrm{C}$, thylakoids were resuspended in a buffer containing $400 \mathrm{mM}$ sorbitol, $15 \mathrm{mM} \mathrm{NaCl}, 5 \mathrm{mM} \mathrm{MgCl}$, and $10 \mathrm{mM} \mathrm{HEPES/KOH,} \mathrm{pH} \mathrm{7.5.}$

In vitro de-epoxidation of violaxanthin. Zeaxanthin (Zea)-containing samples were prepared by in vitro de-epoxidation of the violaxanthin (Vio) in intact thylakoid membranes. Thylakoids were diluted to 50 $\mu \mathrm{g} / \mathrm{mL}$ of chlorophyll (Chl) using a solution containing $330 \mathrm{mM}$ sorbitol, $5 \mathrm{mM} \mathrm{MgCl} 2,10 \mathrm{mM} \mathrm{NaCl}, 40$ $\mathrm{mM}$ MES/NaOH, pH 5.1, $20 \mathrm{mM}$ ascorbate, $0.1 \%(\mathrm{w} / \mathrm{v})$ bovine serum albumin, and protease inhibitors. Thylakoids were stirred for $2 \mathrm{~h}$ at room temperature and then centrifuged at $10,000 \times \mathrm{g}$ for $10 \mathrm{~min}$ at $4^{\circ} \mathrm{C}$.

Purification of LHCII. $3 \mathrm{mg}$ Chl of thylakoid membranes were washed with $5 \mathrm{mM}$ EDTA and resuspended at a final concentration of $1 \mathrm{mg} / \mathrm{mL}$ Chl in $10 \mathrm{mM}$ HEPES, $\mathrm{pH}$ 7.5. Samples were then solubilized at a final concentration of $0.5 \mathrm{mg} / \mathrm{mL} \mathrm{Chl}$, adding $1.6 \% n$-dodecyl $\alpha$-D-maltopyranoside $(\alpha$-DM) and $10 \mathrm{mM}$ HEPES, pH 7.5 and vortexing for $1 \mathrm{~min}$. After $10 \mathrm{~min}$ of incubation on ice, thylakoid membranes were centrifuged at $15,000 \times \mathrm{g}$ for $10 \mathrm{~min}$ to eliminate unsolubilized material. Fractionation occurred upon ultracentrifugation on a $0.1-1 \mathrm{M}$ sucrose gradient containing $0.03 \% \alpha$-DM and $10 \mathrm{mM}$ HEPES, pH 7.5 ( $40 \mathrm{~h}$ at $103,000 \times \mathrm{g}$ at $4^{\circ} \mathrm{C}$ ). The purified LHCII stocks were stored at $-80^{\circ} \mathrm{C}$ until further use, and thawed immediately before each laser measurement.

Pigment composition analysis. The pigments were extracted with $80 \%$ acetone and centrifuged two times at $20,000 \times \mathrm{g}$ for $15 \mathrm{~min}$ at $4{ }^{\circ} \mathrm{C}$. The supernatant was analyzed by HPLC as described in Gilmore $e t$ al. ${ }^{2}$ The pigment composition of the purified LHCII determined from HPLC analysis is shown in Table S1. 
Table S1: Pigment composition of purified LHCII employed in nanodisc reactions. ${ }^{\text {a }}$

\begin{tabular}{c|c|c}
\hline Pigment & LHCII, Vio & LHCII, Zea \\
\hline Chl $a /$ Chl $b$ & $1.28 \pm 0.03$ & $1.30 \pm 0.03$ \\
Chl $a$ per mol & $7.8 \pm 0.08$ & $7.9 \pm 0.07$ \\
Chl $b$ per mol & $6.1 \pm 0.08$ & $6.1 \pm 0.04$ \\
Neo & $0.94 \pm 0.09$ & $1.35 \pm 0.06$ \\
Vio & $0.61 \pm 0.01$ & $0.11 \pm 0.01$ \\
Lut & $2.06 \pm 0.05$ & $2.14 \pm 0.05$ \\
Zea & 0 & $0.67 \pm 0.01$ \\
Chl/Car & $3.88 \pm 0.15$ & $3.28 \pm 0.02$ \\
Chl total & 14 & 14 \\
Car total & $3.6 \pm 0.14$ & $4.3 \pm 0.10$ \\
\hline
\end{tabular}

${ }^{\text {a }}$ Each entry represents the mean \pm std. dev. from four measurements.

\subsubsection{Preparation and characterization of LHCII nanodiscs}

Purification of membrane scaffold protein ApoE422K. ApoE422K, the N-terminal $22 \mathrm{kDa}$ fragment of human apolipoprotein E4, was overexpressed and purified following a previously reported protocol with minor modifications. ${ }^{\sqrt[3]{3}}$ Briefly, thioredoxin-ApoE422K-full length fusion protein was overexpressed from the plasmid pD451-SR containing containing a $6 \times$ histidine tag and kanamycin antibiotic resistance (DNA 2.0), and purified with nickel affinity chromatography. After nickel affinity purification, thrombin (MilliporeSigma) was added to the eluate for cleavage of thioredoxin, and the mixture was incubated overnight at room temperature. SDS-PAGE was used to monitor the progress of the cleavage reaction. Following complete cleavage, the product was purified once again with nickel affinity chromatography, concentrated to $\sim 200 \mu \mathrm{M}$, and aliquoted. Protein concentration was determined using the absorbance of the protein at $280 \mathrm{~nm}$ and the extinction coefficient of ApoE422K at this wavelength $\left(30,940 \mathrm{M}^{-1} \mathrm{~cm}^{-1}\right)$. The aliquots were flash frozen in liquid nitrogen and stored at $-80^{\circ} \mathrm{C}$ until further use.

Preparation of lipid stocks. Preparation of lipids was performed similarly to the method reported in earlier work. ${ }^{[5]}$ Asolectin lipid from soybean was purchased from MilliporeSigma and used without further purification instead of native thylakoid lipid mixture ${ }^{6}$ due to its availability in large quantities as well as the independence of photophysics on the type of lipid used ${ }^{7]} \mathrm{A} 25 \mathrm{mg} / \mathrm{mL}$ of lipid stock solution was prepared by solubilizing asolectin in $50 \mathrm{mM}$ HEPES, $100 \mathrm{mM} \mathrm{NaCl}, 40 \mathrm{mM}$ sodium cholate (pH 7.5). The stock solution was aliquoted, degassed of oxygen, flash frozen and stored at $-80^{\circ} \mathrm{C}$ until further use.

Assembly of the nanodiscs. Nanodiscs loaded with LHCII were produced following a previously reported 
Table S2: Stoichiometry of the nanodisc reactions (per disc).

\begin{tabular}{c|cccc}
\hline$\left\langle N_{\text {LHCII }}\right\rangle$ & 1 & 2 & 3 & 4 \\
\hline LHCII & 1 & 2 & 3 & 4 \\
ApoE422K & 6 & 6 & 6 & 6 \\
Lipid & 1,191 & $1,099.8$ & $1,009.7$ & 918.9 \\
\hline
\end{tabular}

protocol with minor modifications ${ }^{4 \mid 5}$ ] 7 Purified ApoE422K, soy asolectin lipid, and detergent-solubilized LHCII were mixed together at systematically varied molar ratios for production of nanodiscs with $\left\langle N_{\mathrm{LHCII}}\right\rangle$ of 1-4 per disc (Table S2). The mixture was incubated at $4^{\circ} \mathrm{C}$ shaking for $1 \mathrm{~h}$. Detergent was removed by incubating the mixture with Bio-Beads SM-2 adsorbents (Bio-Rad) at $4^{\circ} \mathrm{C}$ for $2 \mathrm{~h}$. The product was purified by the $6 \times$ histidine tag of ApoE422K on a nickel affinity column at $4{ }^{\circ} \mathrm{C}$. Following equilibration of the sample with the beads for $3 \mathrm{~h}$ on a nutating mixer, column flow-through was collected and the column was washed with $10 \mathrm{mM}$ HEPES, $20 \mathrm{mM} \mathrm{NaCl}, 20 \mathrm{mM}$ imidazole, $\mathrm{pH}$ 7.5. The sample was eluted with $10 \mathrm{mM}$ HEPES, $20 \mathrm{mM} \mathrm{NaCl}, 400 \mathrm{mM}$ imidazole, pH 7.5 in fractions. Fractions containing loaded nanodiscs were determined by SDS-PAGE and linear absorption. Because histidine tags were only present in ApoE422K but not in LHCII, aggregates of LHCIIs not incorporated into the discs were removed in the flow-through and washes. Imidazole in the purified discs was removed by buffer exchange into $10 \mathrm{mM}$

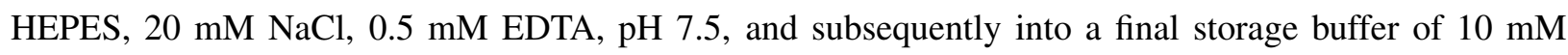
HEPES, $20 \mathrm{mM} \mathrm{NaCl}, \mathrm{pH}$ 7.5. All nanodiscs were first assembled and purified at $\mathrm{pH}$ 7.5. For experiments at low $\mathrm{pH}$, the nanodiscs formed and purified at $\mathrm{pH} 7.5$ were desalted into $10 \mathrm{mM}$ MES, $20 \mathrm{mM} \mathrm{NaCl}(\mathrm{pH}$ 5) using a desalting column (PD-10, GE Healthcare) immediately before each spectroscopic measurement. All spectroscopic measurements were performed within 24 hours of sample preparation. 


\subsection{Imaging and spectroscopic methods}

\subsubsection{Transmission electron microscopy (TEM)}

TEM samples were prepared by the negative staining method ${ }^{8}$ with $2 \%$ uranyl acetate on negatively glowdischarged 400-mesh Cu-carbon coated films (Electron Microscopy Sciences). Samples were imaged on a FEI Tecnai (G2 Spirit TWIN) electron microscope operated at $120 \mathrm{kV}$.

\subsubsection{Steady-state absorption, fluorescence, and circular dichroism (CD)}

Steady-state absorption and fluorescence spectra were measured at $4{ }^{\circ} \mathrm{C}$ with a UV-2401PC spectrophotometer (Shimadzu) and a Cary Eclipse fluorimeter (Agilent), respectively. For fluorescence measurements, the optical density (OD) of the sample at the excitation wavelength $(600 \mathrm{~nm})$ was kept at $0.05-0.07$ to ensure the absence of any reabsorption effect. CD spectra were measured at $4{ }^{\circ} \mathrm{C}$ on a Jasco $\mathrm{J}-1500$ spectropolarimeter. The OD of each sample was 0.2 per mm at $670 \mathrm{~nm}$. All measurements were performed in $1 \mathrm{~cm}$ pathlength quartz cuvettes.

\subsubsection{Time-resolved fluorescence}

Time-resolved fluorescence data were collected with time-correlated single photon counting (TCSPC). The excitation source was generated by coupling the output of a Ti:sapphire oscillator (MaiTai BB, SpectraPhysics; centered at $800 \mathrm{~nm}, 80 \mathrm{MHz}$ repetition rate) into a nonlinear photonic crystal fiber (FemtoWhite 800, NKT Photonics). The generated broadband supercontinuum was then passed through a bandpass filter (ET645/30x, Chroma) to produce the excitation laser spectrum $(630-655 \mathrm{~nm}$, II in Figure S1), and focused into a $100 \mu \mathrm{m}$ spot on a $500 \mu \mathrm{m}$ pathlength flow cuvette. The emission from the sample was first passed through an emission filter (665.2 nm longpass, BLP01-647R-25, Semrock), and then detected by a single-photon-detecting avalanche photodiode (PDM Series, Micro Photon Devices). The arrival time of each photon was detected by a timing module (PicoHarp 300, PicoQuant, Inc.).

The data were measured in two different modes. All of the fluorescence decay traces presented in this work were measured by integrating the signal over the entire spectral range of fluorescence (IV in Figure S1) for a higher signal-to-noise ratio. Spectrally-resolved data were measured with spectral interferometry (Gemini, NIREOS), and then Fourier transformed to yield the time-resolved fluorescence spectra. The resolution of the resultant wavelength axis was $0.35 \mathrm{~nm}$. The interferometer was combined with the photon counting module to also measure fluorescence decay traces at each wavelength. Further details about the spectral interferometry setup and data analysis can be found elsewhere. 9

To prevent reabsorption effect, the OD of all samples at the excitation wavelength range was kept around 0.07 per $500 \mu \mathrm{m}$. The sample solution was continuously flowed with a peristaltic pump during measurement 


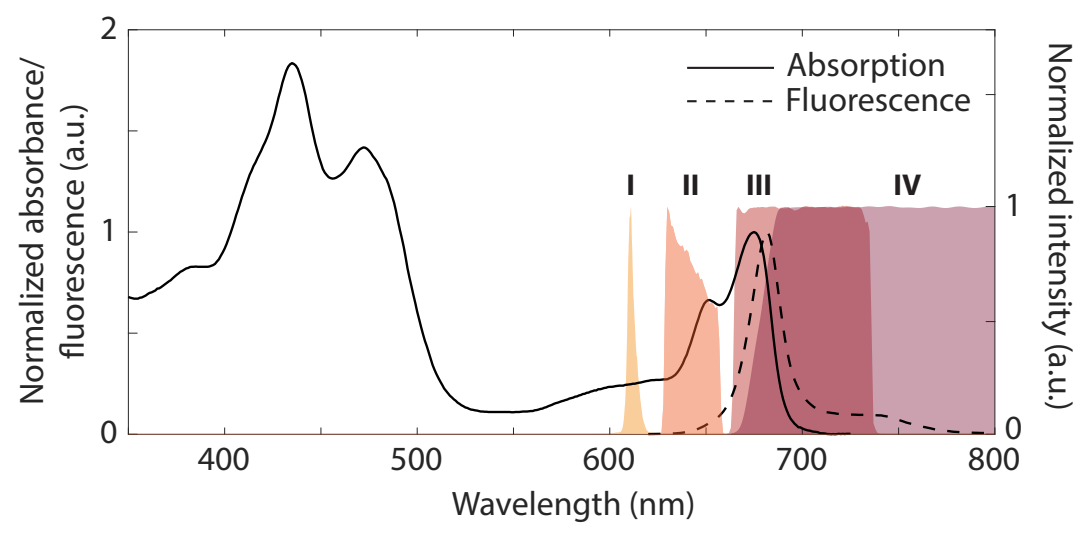

Figure S1: Spectral range of excitation and detection in fluorescence experiments. Black solid and dashed lines show the linear absorption (normalized to the $675 \mathrm{~nm} \mathrm{Chl} a$ peak) and fluorescence (normalized to the $684 \mathrm{~nm}$ fluorescence maximum) spectra of LHCII (in detergent). I and III display the excitation and detection range employed in FCS, respectively. II and IV display the excitation and detection range employed in wavelength-integrated TCSPC, respectively.

to prevent sample photodegradation and thermal lensing effect. Power dependence was checked at three different excitation powers (6 fJ, $20 \mathrm{fJ}$, and $60 \mathrm{fJ}$ ) to ensure the absence of power-dependent artifacts such as singlet-singlet annihilation.10 11 As shown in Figure $\mathrm{S} 10$, no power dependence was observed. The instrument response function (IRF) was determined by measuring scattered excitation light using a dilute solution of colloidal silica (Ludox, MilliporeSigma), $\frac{12}{2}$ and has a width of 50 ps. Fluorescence decay curves were fitted with a mono-exponential or bi-exponential decay function $\left(I(t)=\sum_{n} A_{n} \mathrm{e}^{-t / \tau_{n}}, n=1\right.$ or 2$)$ using iterative reconvolution with the IRF.

\subsubsection{Fluorescence correlation spectroscopy (FCS)}

FCS measurements were performed on a home-built confocal microscope. The excitation source was generated by tuning a fiber laser (FemtoFiber pro, Toptica Photonics; $80 \mathrm{MHz}$ repetition rate), and was centered at $610 \mathrm{~nm}$ with a $4 \mathrm{~nm}$ full-width at half maximum (FWHM) bandwidth (I in Figure S1). The fluorescence signal was isolated using the combination of a dichroic mirror (T635lpxr, Chroma) and a bandpass filter (ET700/75m, Chroma; III in Figure S1]. Samples were diluted to $5 \mathrm{nM}$, and allowed to freely diffuse within a $25 \mathrm{~mm}$ diameter hybridization chamber sealed to a glass coverslip. Sample excitation and fluorescence collection were accomplished by the same oil-immersion objective (UPLSAPO100XO, Olympus; $\mathrm{NA}=1.4)$. The excitation laser fluence on the sample plane was $504 \mathrm{~nJ} / \mathrm{cm}^{2}$. Fluorescence signal was detected using an avalanche photodiode (SPCM-AQRH-15, Excelitas). The diffusion time constant $\left(\tau_{\mathrm{D}}\right)$ was determined by fitting the autocorrelation of the detected photons $\left(G(t)\right.$, typically $2 \times 10^{6}$ photons were 
collected to construct each correlation curve) to equation $(1), 13$

$$
G(t)=C+\left(\frac{1}{N}\right)\left(\frac{1}{1+\frac{t}{\tau_{\mathrm{D}}}}\right)\left(\frac{1}{\sqrt{1+\frac{t}{V^{2} \tau_{\mathrm{D}}}}}\right)\left(1-f_{\mathrm{T}}+f_{\mathrm{T}} \mathrm{e}^{\frac{-t}{\tau_{\mathrm{T}}}}\right)
$$

where $t$ is the correlation time, $C$ is a constant, $N$ is the number of molecules in the focal volume, $\tau_{\mathrm{D}}$ is the diffusion time constant, $V$ is a measure of the detection volume defined as $Z_{o} / w_{o}$, where $Z_{o}$ and $w_{o}$ are the effective half axial and radial dimensions of the focal volume, respectively, $f_{\mathrm{T}}$ is the fractional population of the triplet state, and $\tau_{\mathrm{T}}$ is the triplet lifetime. The diffusion constant $(D)$ and the hydrodynamic radius $(r)$ of each sample were determined using equations (2) and (3) (the Stokes-Einstein relation):

$$
\begin{gathered}
\tau_{\mathrm{D}}=\frac{w_{o}^{2}}{4 D} \\
D=\frac{k_{B} T}{6 \pi \eta r}
\end{gathered}
$$

where $k_{B}$ is the Boltzmann constant, $T$ is the temperature $\left(T=20^{\circ} \mathrm{C}\right.$ was used in this work), and $\eta$ is the viscosity of the medium.

\subsubsection{Ultrabroadband 2D electronic spectroscopy}

A detailed description of the ultrabroadband 2D electronic spectroscopy apparatus can be found elsewhere. 14 Glass filters with different cutoff wavelengths were chosen for each dataset in order to tune the ultrabroadband laser spectrum for optimal excitation of the Car $\mathrm{S}_{2} / \mathrm{Chl} \mathrm{Q}_{\mathrm{x}}$ (spectrum 1) and Chl $\mathrm{Q}_{\mathrm{x}} / \mathrm{Q}_{\mathrm{y}}$ (spectrum 2) regions, respectively (Figure $\$ 2$ a). Spectrum 1 was centered at $550 \mathrm{~nm}\left(18,182 \mathrm{~cm}^{-1}\right)$ with a full-width at half-maximum (FWHM) of $113 \mathrm{~nm}\left(3,819 \mathrm{~cm}^{-1}\right)$, and spectrum 2 was centered at $614 \mathrm{~nm}\left(16,287 \mathrm{~cm}^{-1}\right)$ with a FWHM of $168 \mathrm{~nm}\left(4,807 \mathrm{~cm}^{-1}\right)$. The spectra were compressed with chirped mirror pairs (Ultrafast Innovations $\mathrm{GmbH}$ ) to $6.2-6.9 \mathrm{fs}$ pulses as characterized with transient grating frequency-resolved optical gating (TG-FROG, Figure $\$ 2 \mathrm{~b}, \mathrm{c}$ ) ${ }^{[15}$ 2D electronic spectroscopy was implemented in a BOXCARS geometry with all-parallel polarization. Coherence time $(\tau)$, the time delay between the two pump pulses, was sampled in $0.5 \mathrm{fs}$ steps in the range of -200 to $200 \mathrm{fs}$. Waiting time $(T)$, the time delay between the pump and the probe pulses, was incremented in steps of $20 \mathrm{fs}$ for -200 to $500 \mathrm{fs}, 40 \mathrm{fs}$ for 500 to $980 \mathrm{fs}, 1$ ps for 1 to $10 \mathrm{ps}$, and $10 \mathrm{ps}$ for 10 to $100 \mathrm{ps}$. A pulse energy of $6.3 \mathrm{~nJ}$ was employed for all measurements with a beam waist of $150 \mu \mathrm{m}$ at the sample position, which corresponds to an excitation density of $2.5-2.8 \times 10^{13}$ photons per pulse per $\mathrm{cm}^{2}$, previously reported to be in the linear regime. ${ }^{16}$ All measurements were performed at $4{ }^{\circ} \mathrm{C}$, and the samples were continuously flowed in a $0.5 \mathrm{~mm}$ pathlength flow cuvette with a peristaltic pump to prevent photodegradation and repetitive excitation of the same spot. The OD of the 
samples (measured at $675 \mathrm{~nm}$ ) was 0.2 and 0.15 per $0.5 \mathrm{~mm}$ for the dataset obtained with spectrum 1 and spectrum 2, respectively. The measurements were repeated three times with freshly prepared samples to ensure reproducibility.
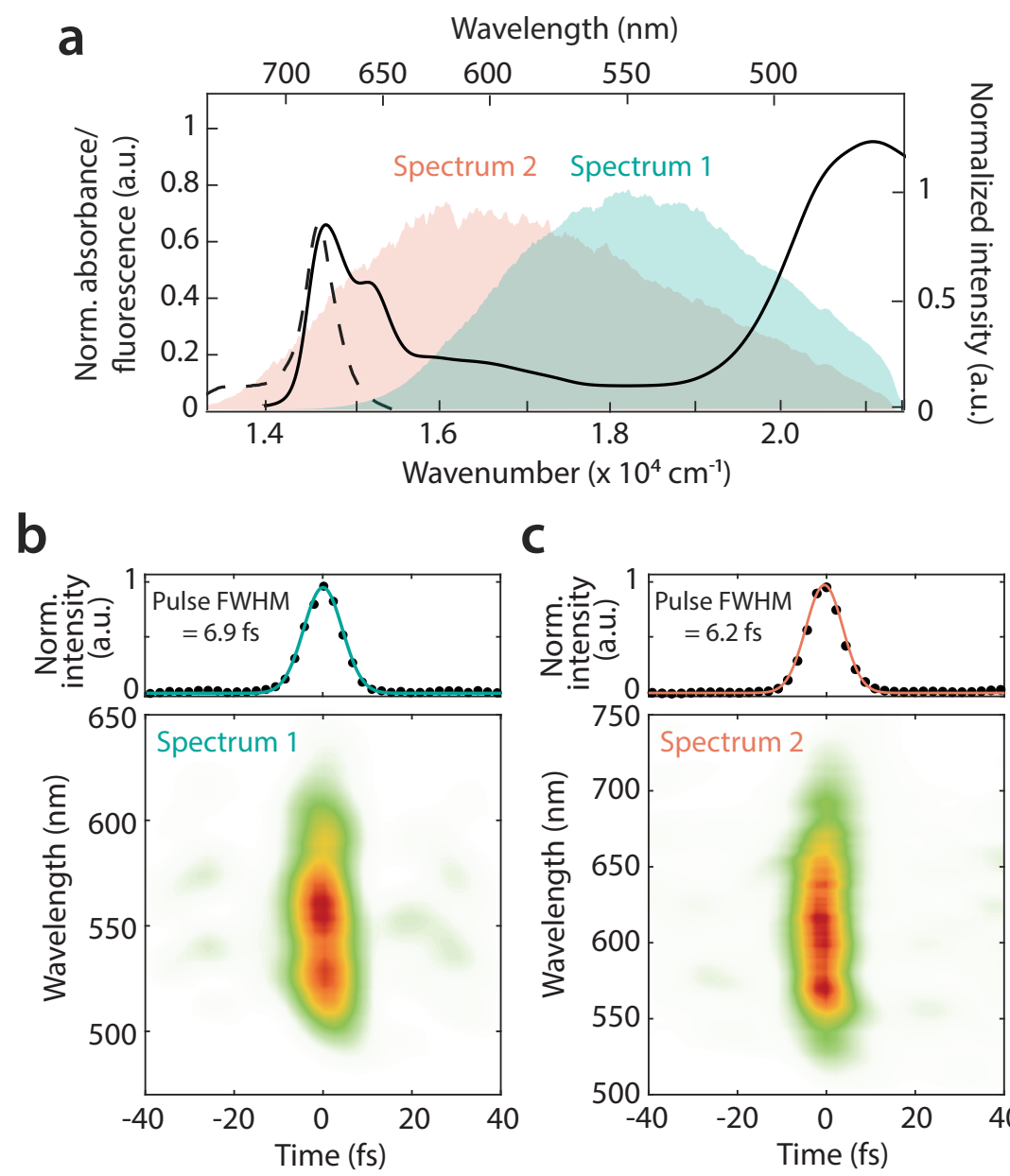

C

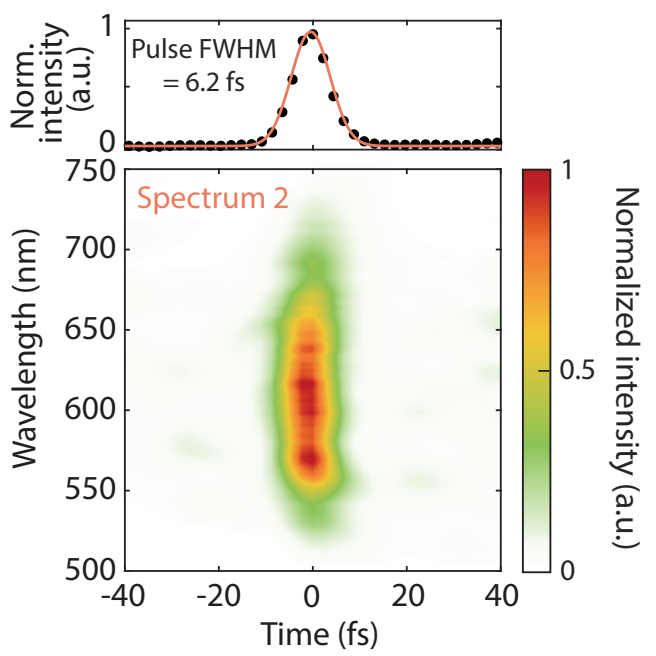

Figure S2: Spectral and temporal profiles of the laser pulses used in 2D electronic spectroscopy. (a)

Ultrabroadband laser spectra used in 2D electronic spectroscopy overlaid with the linear absorption (black solid) and fluorescence spectra (black dashed) of LHCII. (b) - (c), TG-FROG trace of spectrum 1 (b) and spectrum 2 (c) with the retrieved temporal profiles (filled circles) fitted with a Gaussian function (solid line). 


\section{CHARACTERIZATION OF THE NANODISC PRODUCTS}

The purified LHCII nanodiscs were characterized by linear absorption, SDS-PAGE, TEM, and FCS (Figures $\mathrm{S} 3, \mathrm{~S} 4$, and Table $\mathrm{S} 3$. The average LHCII content per disc, $\left\langle N_{\mathrm{LHCII}}\right\rangle$, of the products was confirmed by comparison of the absorbance of the different components of the nanodisc at $280 \mathrm{~nm}$. At this wavelength, all components, i.e., the membrane scaffold protein, lipid, the protein scaffold of the LHCII complex as well as UV absorption of the bound pigments, contribute to the total absorbance. Using the known extinction coefficients for each of the above-mentioned components and the reaction stoichiometry in Table $\$ 2$, we calculated the predicted absorbance at $280 \mathrm{~nm}$ of LHCII $\left(A_{\mathrm{LHCII}}\right)$, which includes contributions from both the protein scaffold and pigments, and of the membrane scaffold protein and the lipid $\left(A_{\mathrm{MSP}+\text { lipid }}\right)$. The ratio of the predicted values $\left(A_{\mathrm{LHCII}} / A_{\mathrm{MSP}+\text { lipid }}\right)$ is shown with a dashed line in Figure $\mathrm{S} 3 \mathrm{~B}$. From the experimental absorption spectra, $A_{\mathrm{LHCII}}$ was extracted based on the visible region of the spectrum, where only the pigments of LHCII absorb. Subtraction of $A_{\mathrm{LHCII}}$ from the total absorbance at $280 \mathrm{~nm}$ yielded the experimental $A_{\mathrm{MSP}+\text { lipid }}$ values, shown with black squares in Figure $\mathrm{S} 3 \mathrm{a}$. The ratios obtained from experimental measurements of the purified nanodiscs are in agreement with the theoretical ratios, verifying successful production of LHCII nanodiscs with the desired $\left\langle N_{\mathrm{LHCII}}\right\rangle$.

The absence of large LHCII aggregates that are unincorporated into the nanodisc was confirmed by FCS. For all $\left\langle N_{\text {LHCII }}\right\rangle$ values, the correlation curves exhibited a single-component decay (Figure $\mathbf{S 4}$ ), whereas a multi-component kinetics is expected in the presence of large, heterogeneous aggregates (Table S(3).
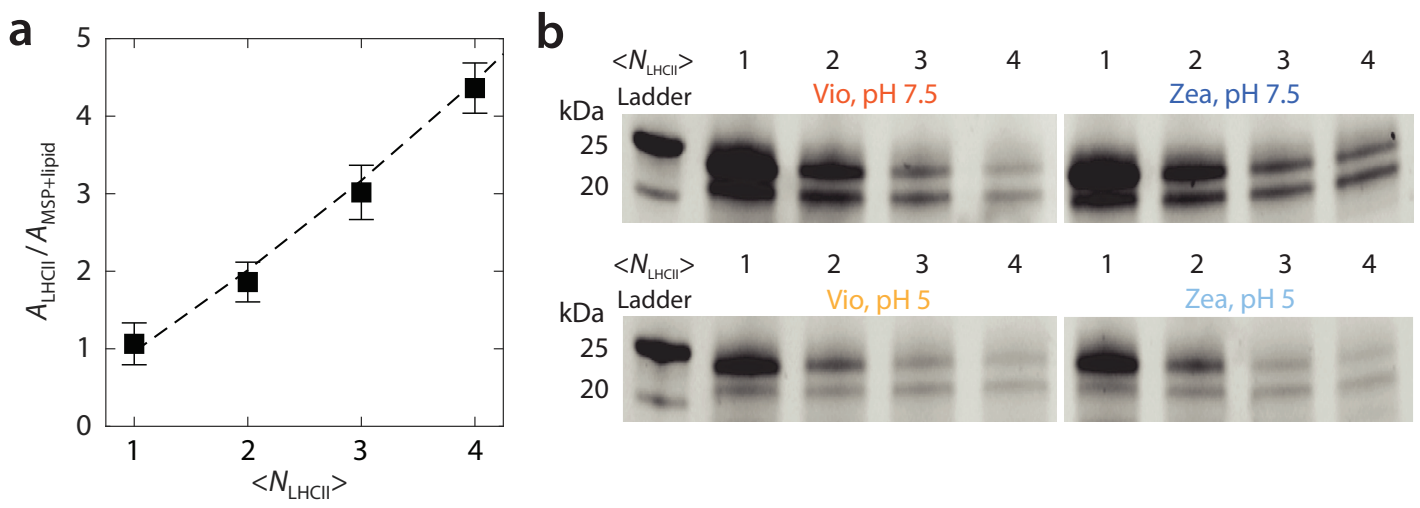

Figure S3: Characterization of the nanodisc products with linear absorption and SDS-PAGE. (a)

Confirmation of the desired stoichiometry based on linear absorption. Dashed curve shows the theoretical ratio, and filled squares are obtained from experimental data. Only the Vio data are shown, but the same trend was observed for Zea. Error bars are std. dev. from five different sample preparations. (b) SDS-PAGE images. Both the ApoE422K (22 kDa) and LHCII bands ( $25 \mathrm{kDa})$ are identified, which shows successful incorporation of LHCII into nanodiscs. 

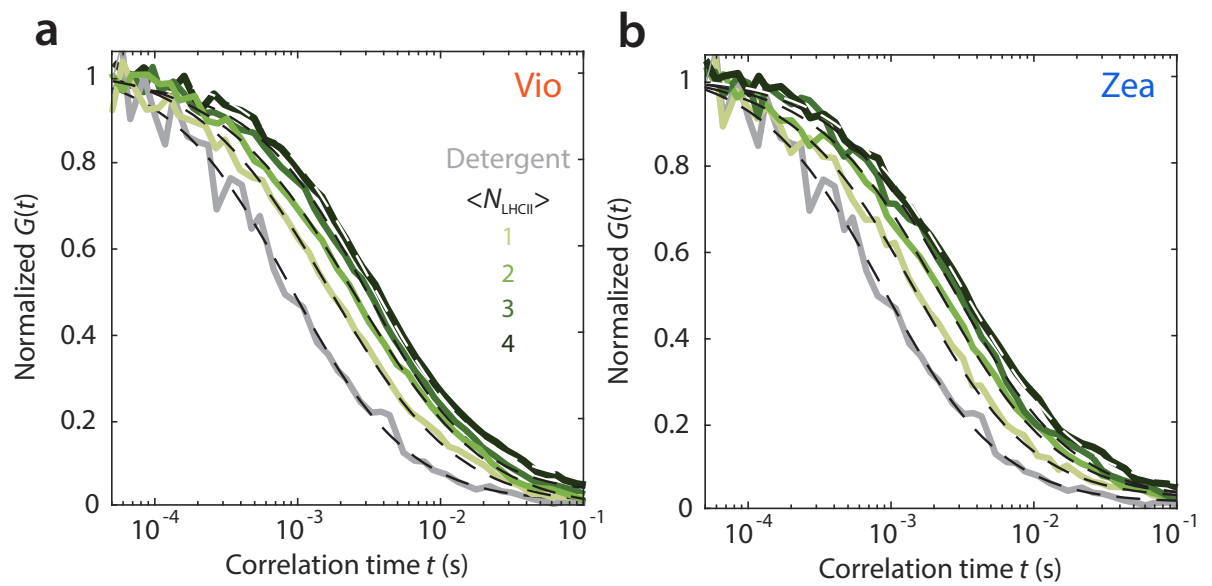

Figure S4: Fluorescence correlation curves. Fluorescence correlation curves of LHCII array nanodiscs as a function of $\left\langle N_{\mathrm{LHCII}}\right\rangle$, with Vio (a) and Zea (b) measured at $\mathrm{pH}$ 7.5. The correlation curves are shown in solid lines, and the fit curves are shown as black/white dashed lines. Gray traces show the correlation curve of LHCII measured in $0.03 \% \alpha$-DM detergent for comparison.

Table S3: Fitted diffusion time constants $\left(\tau_{\mathrm{D}}\right)$, diffusion constants $(D)$, and hydrodynamic radii $(r)$ extracted from the fit. ${ }^{\mathrm{a}}$

\begin{tabular}{|c|c|c|c|c|c|}
\hline & & $\left\langle N_{\text {LHCII }}\right\rangle$ & $\tau_{\mathrm{D}}(\mathrm{ms})$ & $D\left(\mu \mathrm{m}^{2} / \mathrm{s}\right)$ & $r(\mathrm{~nm})$ \\
\hline Detergent & & - & $0.83 \pm 0.06$ & $30.2 \pm 2.3$ & $7.1 \pm 0.5$ \\
\hline \multirow{8}{*}{ Membrane } & \multirow{2}{*}{1} & Vio & $1.76 \pm 0.15$ & $14.3 \pm 1.3$ & $15.1 \pm 1.3$ \\
\hline & & Zea & $1.71 \pm 0.13$ & $14.7 \pm 1.3$ & $14.6 \pm 1.3$ \\
\hline & \multirow{2}{*}{2} & Vio & $2.08 \pm 0.09$ & $12.1 \pm 0.6$ & $17.8 \pm 0.8$ \\
\hline & & Zea & $2.05 \pm 0.10$ & $12.2 \pm 0.6$ & $17.5 \pm 0.7$ \\
\hline & \multirow{2}{*}{3} & Vio & $2.56 \pm 0.08$ & $9.8 \pm 0.3$ & $21.9 \pm 0.7$ \\
\hline & & Zea & $2.50 \pm 0.08$ & $10.0 \pm 0.3$ & $21.4 \pm 0.6$ \\
\hline & \multirow{2}{*}{4} & Vio & $2.92 \pm 0.15$ & $8.6 \pm 0.5$ & $25.0 \pm 1.3$ \\
\hline & & Zea & $2.91 \pm 0.12$ & $8.6 \pm 0.4$ & $24.9 \pm 1.1$ \\
\hline
\end{tabular}

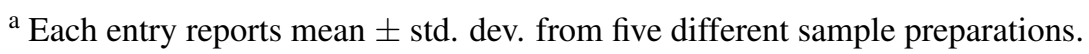




\section{SUPPLEMENTARY DATA}

a

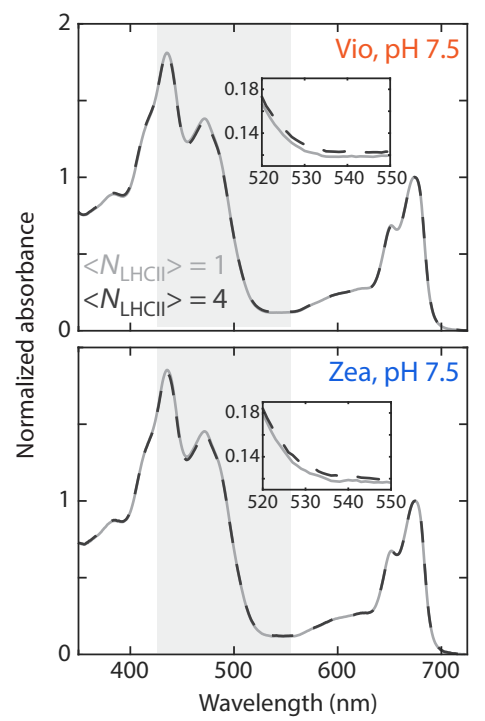

b

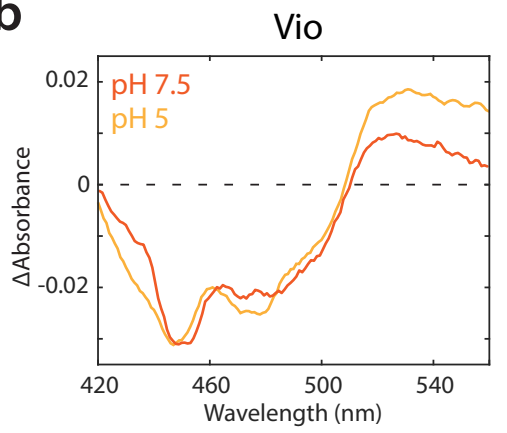

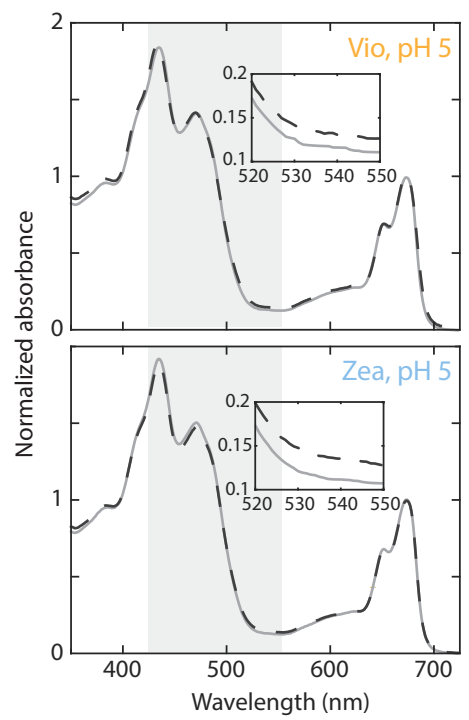

Zea

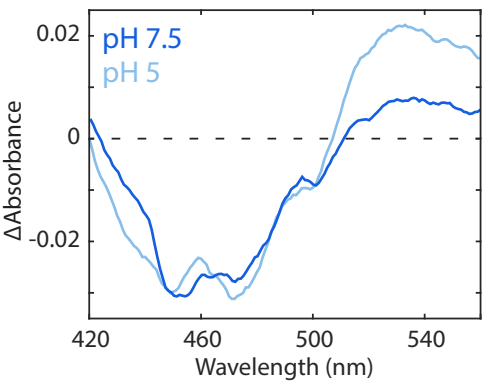

Figure S5: Linear absorption spectra. (a) Linear absorption of the LHCII array nanodiscs shown for

$\left\langle N_{\mathrm{LHCII}}\right\rangle=1$ (gray solid lines) and 4 (black dashed lines). The xanthophyll cycle carotenoid and $\mathrm{pH}$ conditions are indicated in each panel. All spectra are normalized to the Chl $a$ peak at $675 \mathrm{~nm}$. Insets show zoomed-in spectra at 520-550 $\mathrm{nm}$ to better illustrate the increased absorbance in this range upon array formation. (b) Differential absorbance plotted for the carotenoid $S_{2}$ range (gray shaded area in (a)). The spectra are obtained by subtracting the normalized $\left\langle N_{\mathrm{LHCII}}\right\rangle=1$ spectrum from the normalized $\left\langle N_{\mathrm{LHCII}}\right\rangle=$ 4 spectrum, exhibiting the increased absorbance at $535 \mathrm{~nm}$. 

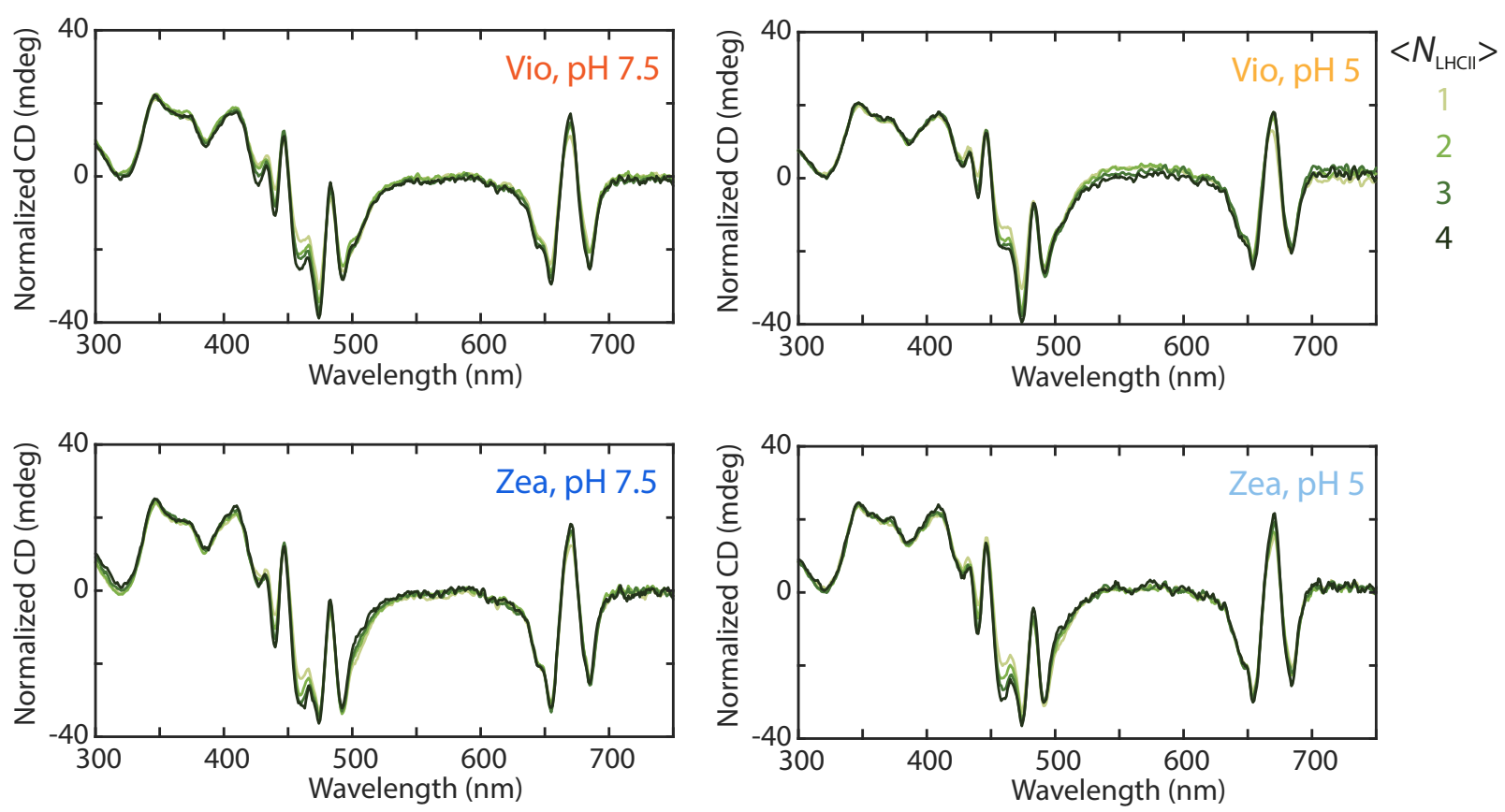

Figure S6: CD spectra. CD spectra of the LHCII array nanodiscs as a function of $\left\langle N_{\mathrm{LHCII}}\right\rangle$. The xanthophyll cycle carotenoid and $\mathrm{pH}$ conditions are indicated in each panel. All spectra are normalized to the sample absorbance at $675 \mathrm{~nm}$.
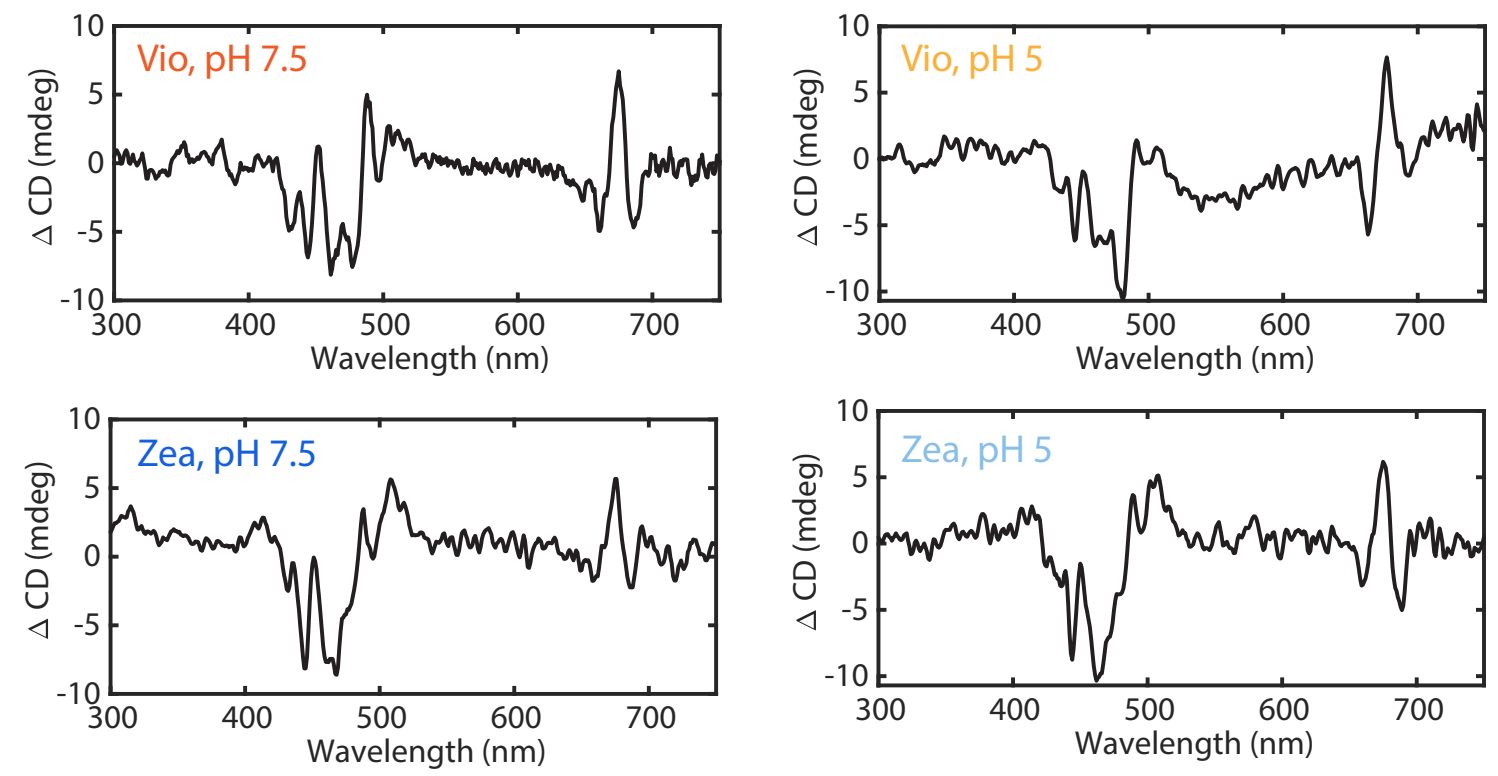

Figure S7: Differential CD spectra. Differential CD $(\Delta C D)$ spectra obtained by subtracting the normalized $\left\langle N_{\mathrm{LHCII}}\right\rangle=1 \mathrm{CD}$ spectrum from that of $\left\langle N_{\mathrm{LHCII}}\right\rangle=4$ nanodiscs. The xanthophyll cycle carotenoid and $\mathrm{pH}$ conditions are indicated in each panel. 

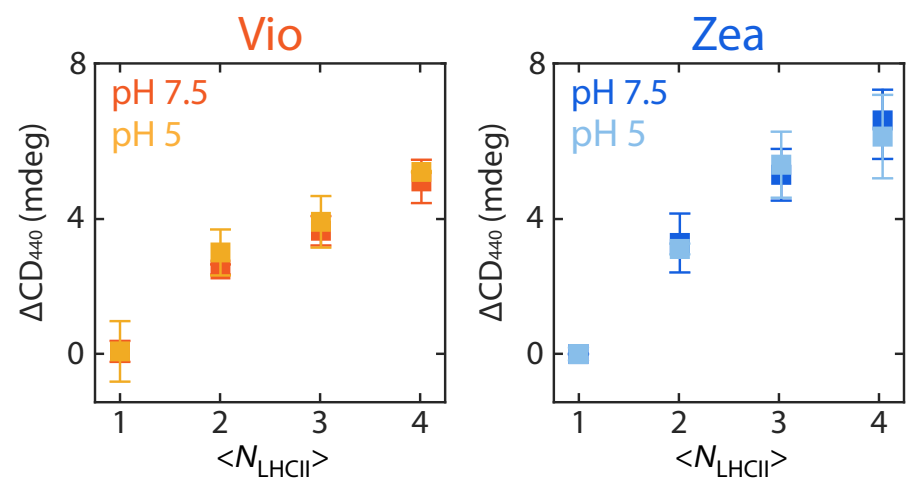

Figure S8: CD intensity change at $440 \mathrm{~nm}$. Differential circular dichroism intensities at $440 \mathrm{~nm}$ $\left(\Delta \mathrm{CD}_{440}\right)$ obtained by subtracting the intensity for $\left\langle N_{\mathrm{LHCII}}\right\rangle=1$ from the respective peak intensity for $\left\langle N_{\mathrm{LHCII}}\right\rangle=2-4$ nanodiscs. The data are plotted as the mean \pm std. dev. of eight independent sample preparations. 

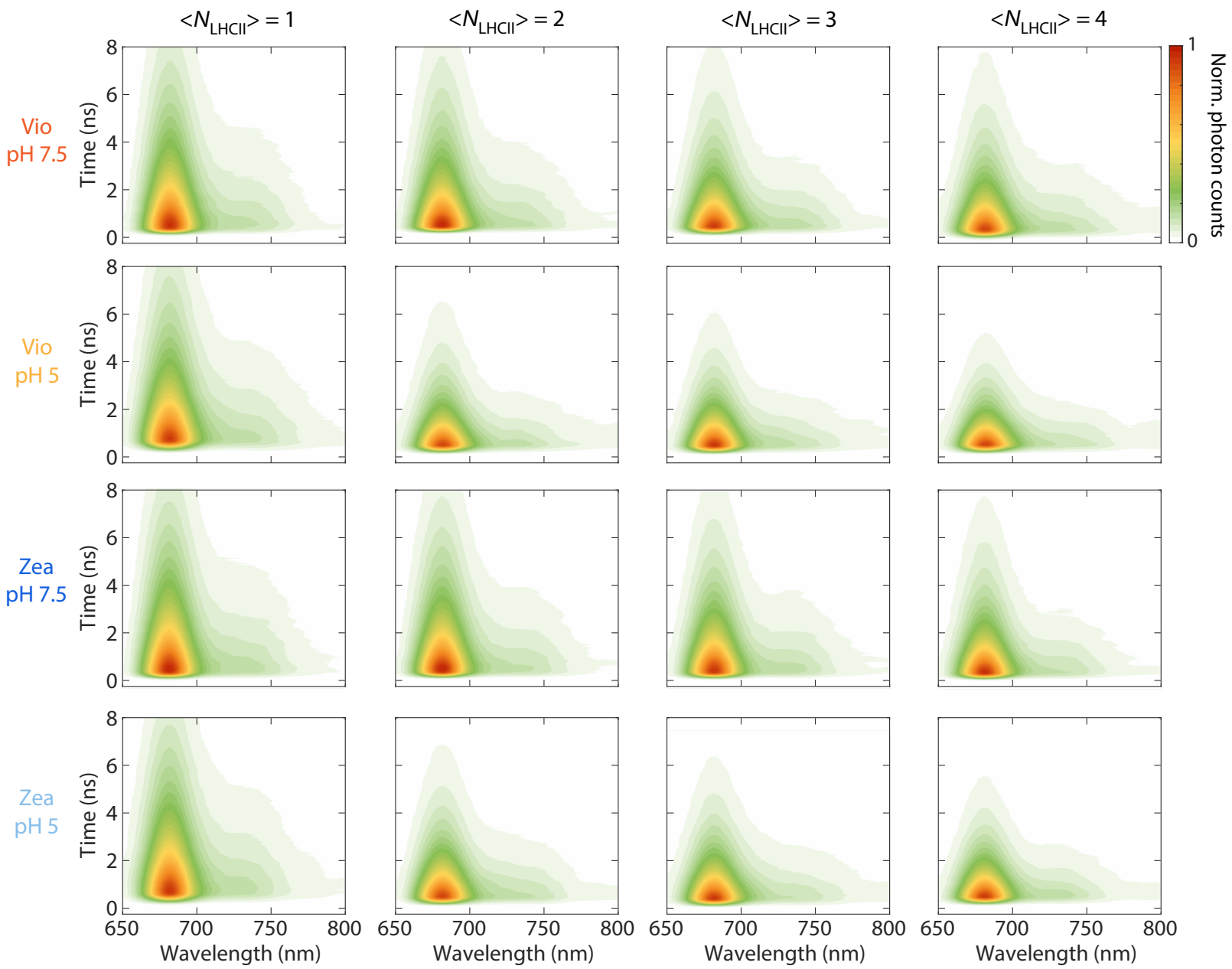

Figure S9: Time-resolved fluorescence spectra. Normalized time-resolved fluorescence spectra of LHCII nanodiscs with $\left\langle N_{\text {LHCII }}\right\rangle=1-4$ with both xanthophyll cycle Car (Vio vs. Zea) and pH (7.5 vs. 5) conditions. 
Table S4: Fit parameters from fluorescence lifetime measurements. ${ }^{a}$

\begin{tabular}{|c|c|c|c|c|c|c|c|}
\hline Car & $\mathrm{pH}$ & $\left\langle N_{\text {LHCII }}\right\rangle$ & $A_{1}(\%)$ & $\tau_{1}(\mathrm{~ns})$ & $A_{2}(\%)$ & $\tau_{2}(\mathrm{~ns})$ & $\left\langle\tau_{\mathrm{ff}}\right\rangle^{\mathrm{b}}$ \\
\hline \multirow{8}{*}{ Vio } & \multirow{4}{*}{7.5} & 1 & $11.0 \pm 1.0$ & $0.30 \pm 0.03$ & $89.0 \pm 0.8$ & $3.15 \pm 0.08$ & $2.84 \pm 0.07$ \\
\hline & & 2 & $23.0 \pm 1.1$ & $0.30 \pm 0.06$ & $77.0 \pm 0.8$ & $2.84 \pm 0.10$ & $2.26 \pm 0.14$ \\
\hline & & 3 & $25.5 \pm 1.2$ & $0.34 \pm 0.06$ & $74.5 \pm 1.0$ & $2.58 \pm 0.11$ & $2.01 \pm 0.12$ \\
\hline & & 4 & $28.7 \pm 1.2$ & $0.35 \pm 0.07$ & $71.3 \pm 1.0$ & $2.44 \pm 0.09$ & $1.84 \pm 0.12$ \\
\hline & \multirow{4}{*}{5} & 1 & $11.8 \pm 0.8$ & $0.29 \pm 0.03$ & $88.2 \pm 0.8$ & $3.10 \pm 0.08$ & $2.77 \pm 0.14$ \\
\hline & & 2 & $26.0 \pm 0.5$ & $0.28 \pm 0.04$ & $74.0 \pm 0.8$ & $2.10 \pm 0.09$ & $1.63 \pm 0.07$ \\
\hline & & 3 & $31.0 \pm 0.6$ & $0.28 \pm 0.06$ & $69.0 \pm 0.7$ & $1.88 \pm 0.05$ & $1.38 \pm 0.06$ \\
\hline & & 4 & $33.0 \pm 0.6$ & $0.28 \pm 0.06$ & $67.0 \pm 0.6$ & $1.76 \pm 0.10$ & $1.27 \pm 0.08$ \\
\hline \multirow{8}{*}{ Zea } & \multirow{4}{*}{7.5} & 1 & $9.7 \pm 1.0$ & $0.29 \pm 0.02$ & $90.3 \pm 0.9$ & $3.09 \pm 0.07$ & $2.82 \pm 0.07$ \\
\hline & & 2 & $22.3 \pm 1.0$ & $0.31 \pm 0.07$ & $77.7 \pm 0.5$ & $2.92 \pm 0.10$ & $2.34 \pm 0.16$ \\
\hline & & 3 & $24.0 \pm 0.8$ & $0.31 \pm 0.07$ & $76.0 \pm 0.9$ & $2.63 \pm 0.11$ & $2.07 \pm 0.15$ \\
\hline & & 4 & $27.0 \pm 0.8$ & $0.32 \pm 0.05$ & $73.3 \pm 0.6$ & $2.50 \pm 0.12$ & $1.91 \pm 0.11$ \\
\hline & \multirow{4}{*}{5} & 1 & $12.0 \pm 0.9$ & $0.28 \pm 0.03$ & $88.0 \pm 0.8$ & $3.06 \pm 0.05$ & $2.73 \pm 0.13$ \\
\hline & & 2 & $24.2 \pm 0.3$ & $0.32 \pm 0.04$ & $75.8 \pm 0.3$ & $2.26 \pm 0.05$ & $1.79 \pm 0.04$ \\
\hline & & 3 & $27.0 \pm 0.7$ & $0.32 \pm 0.03$ & $73.0 \pm 0.8$ & $1.98 \pm 0.07$ & $1.53 \pm 0.06$ \\
\hline & & 4 & $31.0 \pm 0.6$ & $0.30 \pm 0.04$ & $69.0 \pm 0.7$ & $1.84 \pm 0.09$ & $1.37 \pm 0.09$ \\
\hline
\end{tabular}

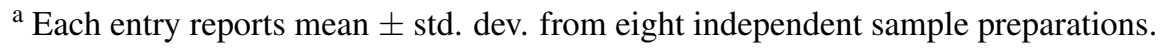

${ }^{\mathrm{b}}$ Amplitude-weighted average fluorescence lifetime $\left(\left\langle\tau_{\mathrm{fl}}\right\rangle=\frac{\sum A_{n} \tau_{n}}{\sum A_{n}}\right)$. 

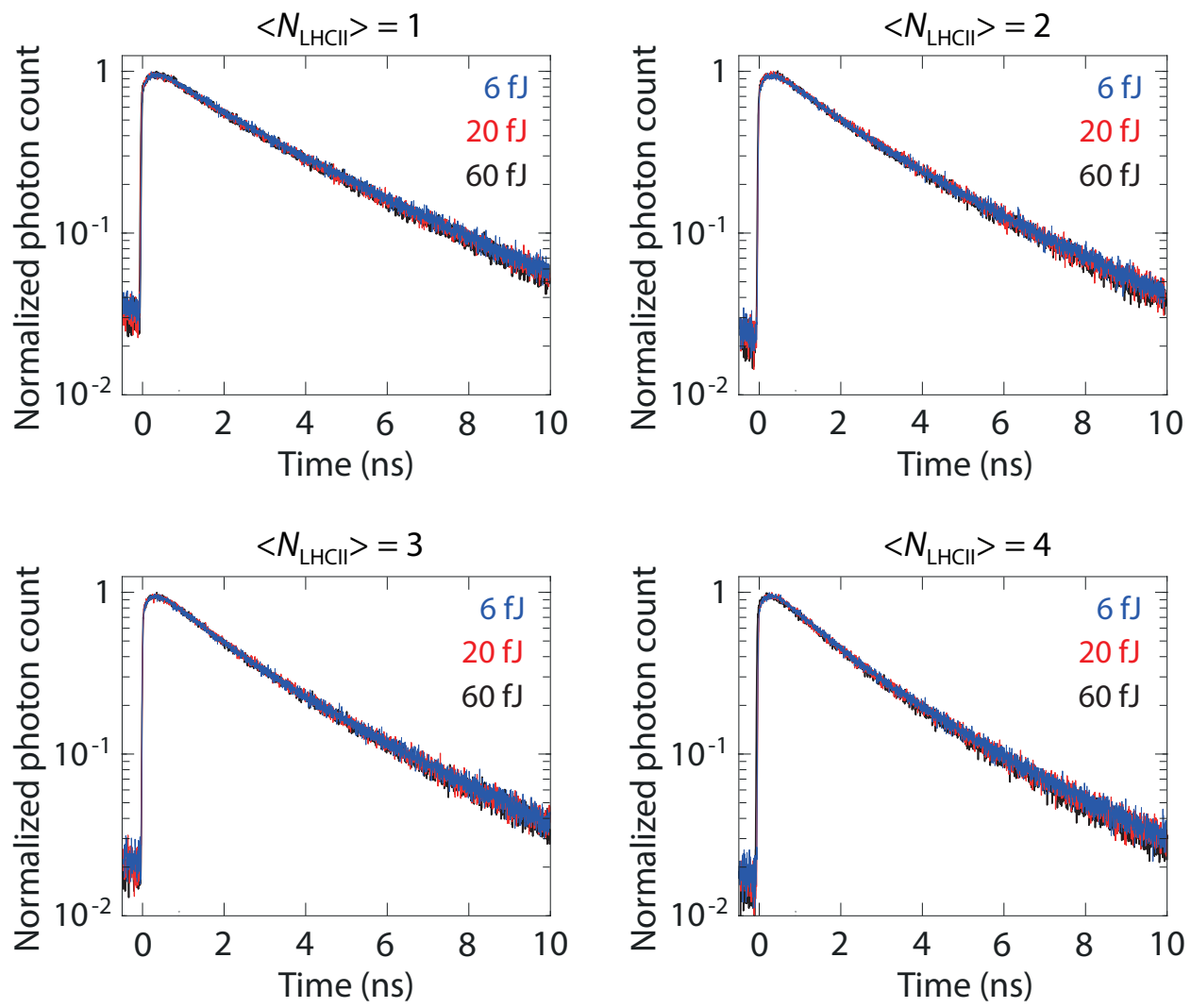

Figure S10: Independence of the fluorescence decay profiles on excitation power. Normalized

fluorescence decay profiles of LHCII array nanodiscs with $\left\langle N_{\mathrm{LHCII}}\right\rangle=1-4$ measured at $6 \mathrm{fJ}$ (blue), $20 \mathrm{fJ}$ (red), and $60 \mathrm{fJ}$ (black), confirming the absence of power dependence. The data are shown for Zea at $\mathrm{pH}$ 7.5 only, but the same trend was observed for Vio and at $\mathrm{pH} 5$. 

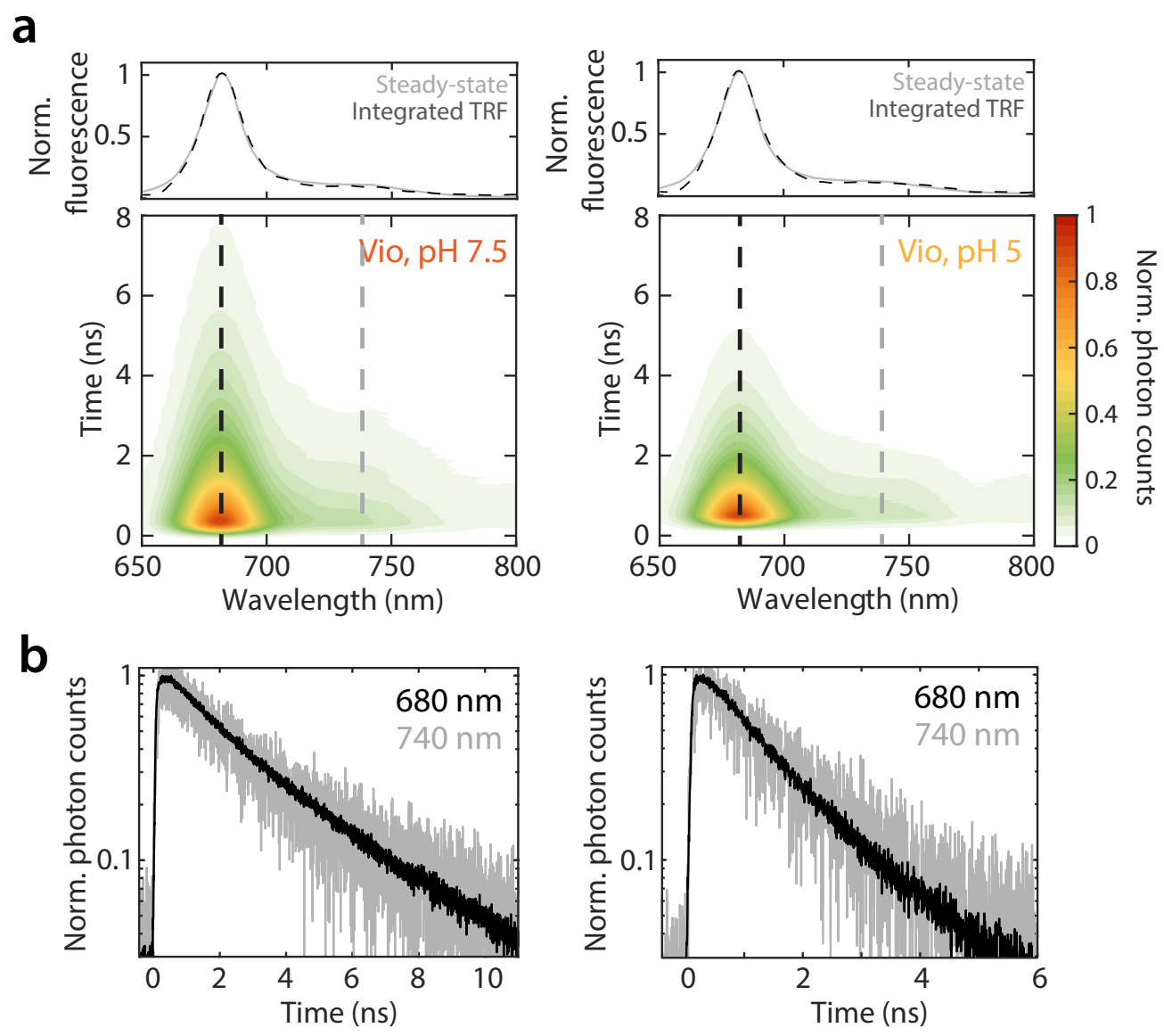

Figure S11: Wavelength independence of the fluorescence decay. (a) Time-resolved fluorescence spectra of $\left\langle N_{\mathrm{LHCII}}\right\rangle=4$ nanodiscs with Vio, at $\mathrm{pH} 7.5$ (left) and $\mathrm{pH} 5$ (right). The top panels show overlays of the steady-state fluorescence spectrum (gray solid line) and temporally integrated time-resolved fluorescence spectrum (black dashed line), demonstrating the agreement between the two spectra. The black and gray dashed lines indicate the wavelengths at which the traces in (b) were generated. (b) Normalized fluorescence decay profiles at $680 \mathrm{~nm}$ (black) and gray $(740 \mathrm{~nm}$ ) overlaid for the $\mathrm{pH} 7.5$ (left) and $\mathrm{pH} 5$ (right) maps, showing independence of the dynamics of emission wavelength. 

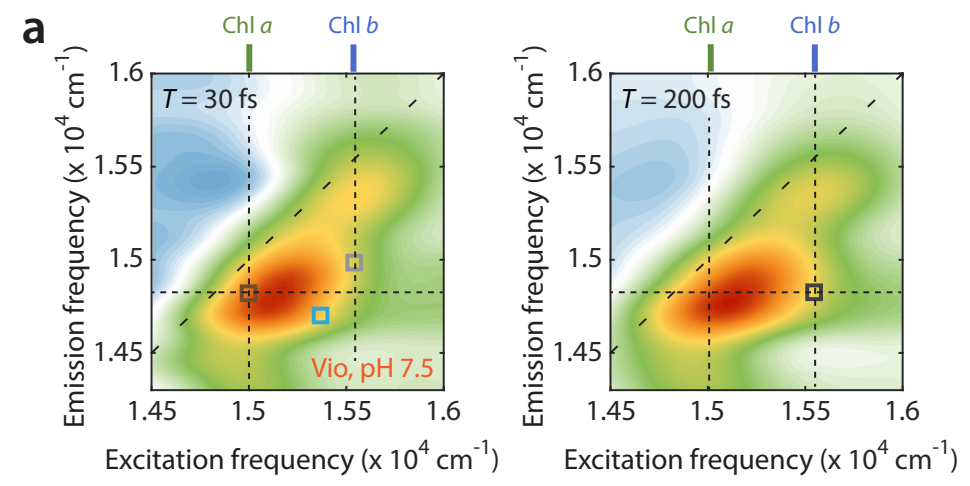

b Background-subtracted

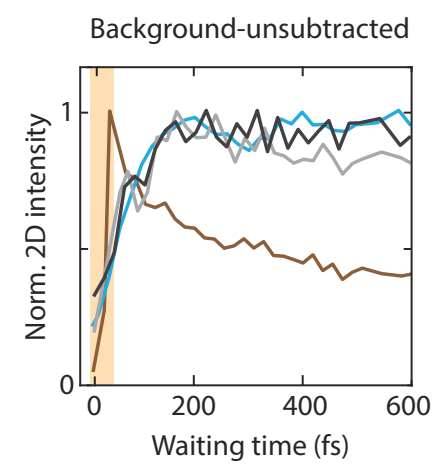

Figure S12: Chlorophyll energy transfer cross peaks at early waiting times. (a) 2D spectra of $\left\langle N_{\mathrm{LHCII}}\right\rangle$ $=4$ nanodiscs (Vio, pH 7.5) at $T=30 \mathrm{fs}$ (left) and $200 \mathrm{fs}$ (right). (b) Normalized waiting time traces generated at three different diagonal and cross peak positions (labeled with colored squares in (a)). The trace shown in Figure $3 b$ of the main text is plotted in black. The $\sim 60$ fs delayed growth is only present for the cross peaks, whereas the chlorophyll $a$ diagonal peak (brown) rises instantaneously. The three cross peak positions probed showed identical dynamics. Left plot shows normalized traces after background subtraction, such that all peaks are at zero intensity at $T=0$ fs. Right plot shows the background-unsubtracted data, illustrating the cross peak intensity is $20-30 \%$ of the maximum at $T=0 \mathrm{fs}$ (highlighted with orange shaded region). 

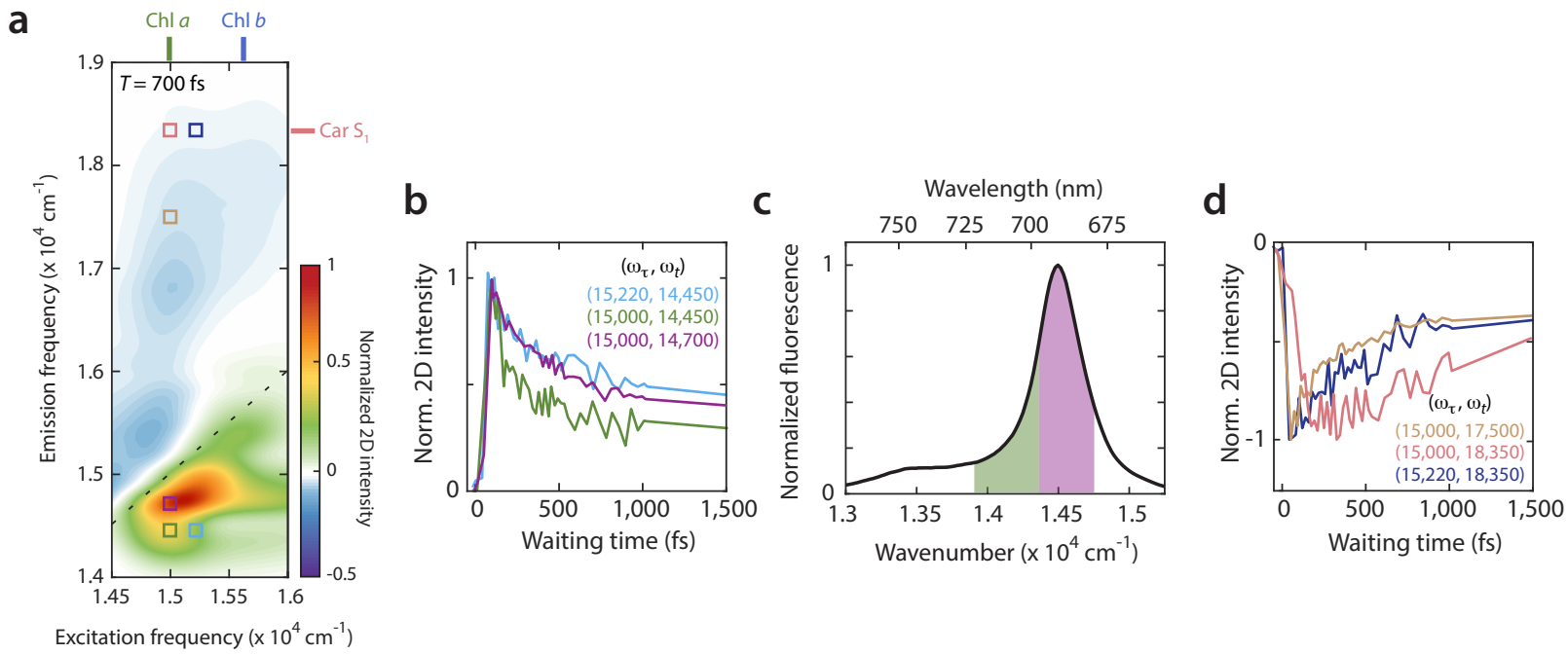

Figure S13: Frequency dependence of the low-energy chlorophyll $a$ and carotenoid $S_{1}$ ESA features.

(a) 2D spectrum of $\left\langle N_{\mathrm{LHCII}}\right\rangle=4$ nanodiscs (Vio, pH 7.5) at $T=700 \mathrm{fs}$ (same as Figure 3a in the main text). Colored squares indicate the peak positions where the traces shown in (b) and (d) were generated. (b) Comparison of the normalized low-energy chlorophyll $a$ waiting time traces at frequencies labeled in (a) and indicated in the parentheses ( $\omega_{\tau}$ : excitation frequency, $\omega_{t}$ : emission frequency). (c) Steady-state fluorescence spectrum labeling the low-energy (green) and high-energy (purple) chlorophyll $a$ ranges.

Rapid dissipative decay was observed only when the low-energy range was probed. (d) Comparison of the normalized carotenoid $\mathrm{S}_{1}$ ESA waiting time traces at frequencies labeled in (a) and indicated in the parentheses. In (b) and (d), all traces were generated by integrating over a $100 \mathrm{~cm}^{-1} \omega_{\tau} \times 100 \mathrm{~cm}^{-1} \omega_{t}$ range around the center frequencies indicated in the plots. 
a

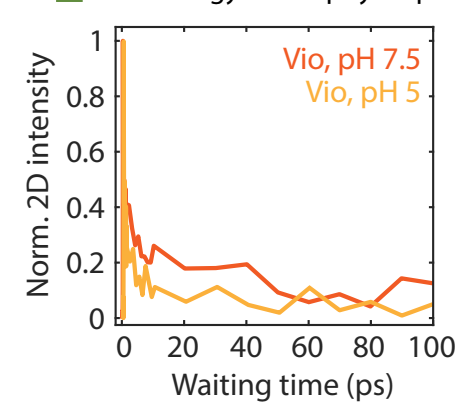

b

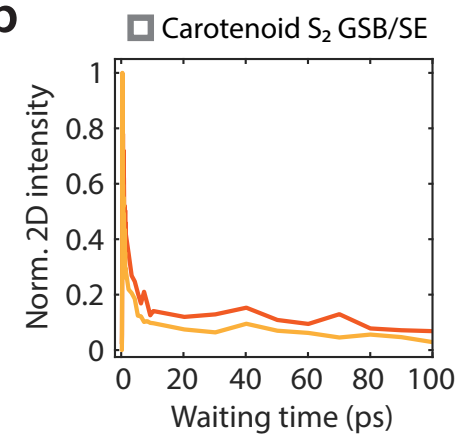

$\square$ Chlorophyll a/carotenoid $\mathrm{S}_{1}$ cross peak
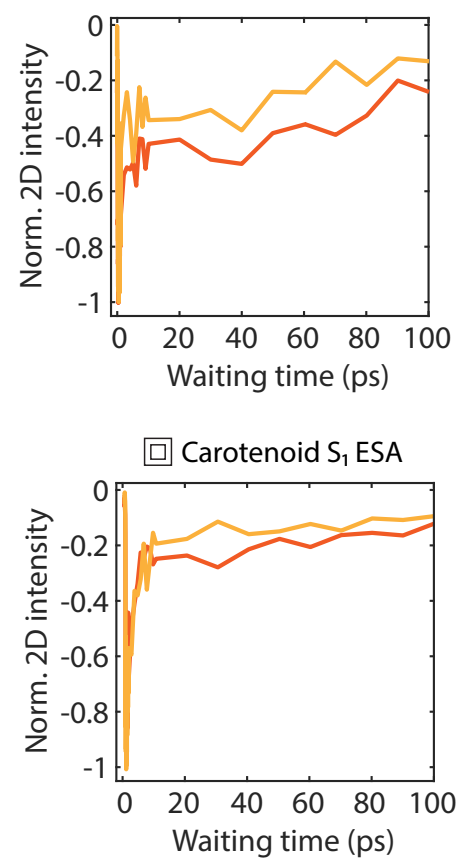

Figure S14: Longer-timescale dissipative dynamics. (a) and (b) show the full time traces of the traces shown in Figures $3 \mathrm{c}$ and Figure $4 \mathrm{~b}, \mathrm{c}$ in the main text, respectively, for $\left\langle N_{\mathrm{LHCII}}\right\rangle=4$ nanodiscs (Vio). All traces are normalized to the maximum (minimum) 2D intensity for positive (negative) features. 


\section{TABLES OF FIT PARAMETERS FROM 2D ELECTRONIC SPECTROSCOPY MEASUREMENTS}

In all tables in this section, each entry reports the $95 \%$ confidence interval from fitting to a multiexponential function convolved with the time resolution of the $2 \mathrm{D}$ electronic spectroscopy measurement (Figure S2). A reports the normalized percent amplitude for each component, where positive and negative amplitudes indicate exponential decay and rise, respectively. $t$ reports the fitted time constant for each component.

Table S5: Fit parameters of the chlorophyll $b$ peak

\begin{tabular}{c|c|cccccc}
\hline & & $A_{1}(\%)$ & $t_{1}(\mathrm{fs})$ & $A_{2}(\%)$ & $t_{2}(\mathrm{fs})$ & $A_{3}(\%)$ & $t_{3}(\mathrm{fs})$ \\
\hline \multirow{2}{*}{ Vio } & $\left\langle N_{\mathrm{LHCII}}\right\rangle=1$ & - & - & $53 \pm 5$ & $370 \pm 35$ & $47 \pm 5$ & $3600 \pm 390$ \\
& $\left\langle N_{\mathrm{LHCII}}\right\rangle=4, \mathrm{pH} 7.5$ & $70 \pm 4$ & $55 \pm 12$ & $16 \pm 4$ & $360 \pm 30$ & $14 \pm 4$ & $10340 \pm 5500$ \\
& $\left\langle N_{\mathrm{LHCII}}\right\rangle=4, \mathrm{pH} 5$ & $69 \pm 5$ & $49 \pm 15$ & $19 \pm 5$ & $347 \pm 30$ & $12 \pm 5$ & $9150 \pm 5000$ \\
\hline \multirow{2}{*}{ Zea } & $\left\langle N_{\mathrm{LHCII}}\right\rangle=1$ & - & - & $55 \pm 5$ & $380 \pm 45$ & $45 \pm 5$ & $3500 \pm 400$ \\
& $\left\langle N_{\mathrm{LHCII}}\right\rangle=4, \mathrm{pH} 7.5$ & $68 \pm 4$ & $58 \pm 18$ & $19 \pm 4$ & $390 \pm 40$ & $13 \pm 4$ & $10200 \pm 4000$ \\
& $\left\langle N_{\mathrm{LHCII}}\right\rangle=4, \mathrm{pH} 5$ & $70 \pm 5$ & $48 \pm 12$ & $20 \pm 5$ & $340 \pm 30$ & $10 \pm 5$ & $8030 \pm 4700$ \\
\hline
\end{tabular}

Table S6: Fit parameters of the chlorophyll $b$-chlorophyll $a$ cross peak

\begin{tabular}{c|c|cccc}
\hline & & $A_{1}(\%)$ & $t_{1}(\mathrm{fs})$ & $A_{2}(\%)$ & $t_{2}(\mathrm{fs})$ \\
\hline \multirow{2}{*}{ Vio } & $\left\langle N_{\mathrm{LHCII}}\right\rangle=1$ & $-36 \pm 5$ & $225 \pm 20$ & $64 \pm 5$ & $8600 \pm 1700$ \\
& $\left\langle N_{\mathrm{LHCII}}\right\rangle=4, \mathrm{pH} 7.5$ & $-49 \pm 4$ & $59 \pm 7$ & $51 \pm 4$ & $5660 \pm 600$ \\
& $\left\langle N_{\mathrm{LHCII}}\right\rangle=4, \mathrm{pH} 5$ & $-50 \pm 5$ & $57 \pm 6$ & $50 \pm 5$ & $5450 \pm 650$ \\
\hline \multirow{2}{*}{ Zea } & $\left\langle N_{\mathrm{LHCII}}\right\rangle=1$ & $-55 \pm 5$ & $220 \pm 25$ & $45 \pm 5$ & $8550 \pm 1800$ \\
& $\left\langle N_{\mathrm{LHCII}}\right\rangle=4, \mathrm{pH} 7.5$ & $-50 \pm 4$ & $55 \pm 8$ & $50 \pm 4$ & $5500 \pm 900$ \\
& $\left\langle N_{\mathrm{LHCII}}\right\rangle=4, \mathrm{pH} 5$ & $-49 \pm 5$ & $58 \pm 5$ & $51 \pm 5$ & $5650 \pm 650$ \\
\hline
\end{tabular}

Table S7: Fit parameters of the chlorophyll $a$-carotenoid $S_{1}$ ESA cross peak

\begin{tabular}{c|c|cccc}
\hline & & $A_{1}(\%)$ & $t_{1}(\mathrm{fs})$ & $A_{2}(\%)$ & $t_{2}(\mathrm{fs})$ \\
\hline \multirow{3}{*}{ Vio } & $\left\langle N_{\text {LHCII }}\right\rangle=1$ & $-41 \pm 7$ & $240 \pm 25$ & $59 \pm 7$ & $4100 \pm 700$ \\
& $\left\langle N_{\text {LHCII }}\right\rangle=4, \mathrm{pH} 7.5$ & $-38 \pm 4$ & $150 \pm 30$ & $62 \pm 4$ & $1520 \pm 480$ \\
& $\left\langle N_{\text {LHCII }}\right\rangle=4, \mathrm{pH} 5$ & $-45 \pm 4$ & $150 \pm 20$ & $55 \pm 4$ & $1160 \pm 400$ \\
\hline \multirow{2}{*}{ Zea } & $\left\langle N_{\mathrm{LHCII}}\right\rangle=1$ & $-43 \pm 5$ & $236 \pm 25$ & $57 \pm 5$ & $4480 \pm 1000$ \\
& $\left\langle N_{\mathrm{LHCII}}\right\rangle=4, \mathrm{pH} 7.5$ & $-40 \pm 4$ & $150 \pm 20$ & $60 \pm 4$ & $1630 \pm 450$ \\
& $\left\langle N_{\mathrm{LHCII}}\right\rangle=4, \mathrm{pH} 5$ & $-48 \pm 5$ & $150 \pm 35$ & $52 \pm 5$ & $1245 \pm 500$ \\
\hline
\end{tabular}


Table S8: Fit parameters of the low-energy chlorophyll $a$ peak

\begin{tabular}{c|c|cccc}
\hline & & $A_{1}(\%)$ & $t_{1}(\mathrm{fs})$ & $A_{2}(\%)$ & $t_{2}(\mathrm{fs})$ \\
\hline \multirow{2}{*}{ Vio } & $\left\langle N_{\text {LHCII }}\right\rangle=1$ & $49 \pm 5$ & $255 \pm 30$ & $51 \pm 5$ & $4300 \pm 1200$ \\
& $\left\langle N_{\text {LHCII }}\right\rangle=4, \mathrm{pH} 7.5$ & $63 \pm 5$ & $120 \pm 30$ & $37 \pm 5$ & $8200 \pm 2800$ \\
& $\left\langle N_{\text {LHCII }}\right\rangle=4, \mathrm{pH} 5$ & $82 \pm 4$ & $118 \pm 20$ & $18 \pm 4$ & $8700 \pm 1900$ \\
\hline \multirow{2}{*}{ Zea } & $\left\langle N_{\mathrm{LHCII}}\right\rangle=1$ & $48 \pm 5$ & $255 \pm 25$ & $52 \pm 5$ & $4250 \pm 900$ \\
& $\left\langle N_{\mathrm{LHCII}}\right\rangle=4, \mathrm{pH} 7.5$ & $68 \pm 4$ & $122 \pm 30$ & $32 \pm 4$ & $8330 \pm 2200$ \\
& $\left\langle N_{\mathrm{LHCII}}\right\rangle=4, \mathrm{pH} 5$ & $79 \pm 5$ & $108 \pm 20$ & $21 \pm 5$ & $9100 \pm 2000$ \\
\hline
\end{tabular}

Table S9: Fit parameters of the carotenoid $S_{2}$ GSB/SE peak

\begin{tabular}{c|c|cccc}
\hline & & $A_{1}(\%)$ & $t_{1}(\mathrm{fs})$ & $A_{2}(\%)$ & $t_{2}(\mathrm{fs})$ \\
\hline \multirow{2}{*}{ Vio } & $\left\langle N_{\mathrm{LHCII}}\right\rangle=1$ & $39 \pm 5$ & $150 \pm 20$ & $61 \pm 5$ & $5000 \pm 1700$ \\
& $\left\langle N_{\mathrm{LHCII}}\right\rangle=4, \mathrm{pH} 7.5$ & $38 \pm 5$ & $165 \pm 20$ & $62 \pm 5$ & $5200 \pm 1500$ \\
& $\left\langle N_{\mathrm{LHCII}}\right\rangle=4, \mathrm{pH} 5$ & $50 \pm 4$ & $165 \pm 20$ & $50 \pm 4$ & $4700 \pm 1900$ \\
\hline \multirow{2}{*}{ Zea } & $\left\langle N_{\mathrm{LHCII}}\right\rangle=1$ & $38 \pm 5$ & $155 \pm 25$ & $62 \pm 5$ & $4950 \pm 1900$ \\
& $\left\langle N_{\mathrm{LHCII}}\right\rangle=4, \mathrm{pH} 7.5$ & $40 \pm 6$ & $165 \pm 20$ & $60 \pm 6$ & $6300 \pm 2200$ \\
& $\left\langle N_{\mathrm{LHCII}}\right\rangle=4, \mathrm{pH} 5$ & $49 \pm 5$ & $165 \pm 20$ & $51 \pm 5$ & $5100 \pm 2400$ \\
\hline
\end{tabular}

Table S10: Fit parameters of the carotenoid $S_{1}$ ESA peak

\begin{tabular}{c|c|cccc}
\hline & & $A_{1}(\%)$ & $t_{1}(\mathrm{fs})$ & $A_{2}(\%)$ & $t_{2}(\mathrm{fs})$ \\
\hline \multirow{2}{*}{ Vio } & $\left\langle N_{\mathrm{LHCII}}\right\rangle=1$ & $-39 \pm 5$ & $142 \pm 28$ & $61 \pm 5$ & $5300 \pm 1700$ \\
& $\left\langle N_{\mathrm{LHCII}}\right\rangle=4, \mathrm{pH} 7.5$ & $-47 \pm 4$ & $165 \pm 20$ & $53 \pm 4$ & $4520 \pm 1500$ \\
& $\left\langle N_{\mathrm{LHCII}}\right\rangle=4, \mathrm{pH} 5$ & $-50 \pm 4$ & $165 \pm 20$ & $50 \pm 4$ & $4300 \pm 1150$ \\
\hline \multirow{2}{*}{ Zea } & $\left\langle N_{\mathrm{LHCII}}\right\rangle=1$ & $-38 \pm 5$ & $146 \pm 29$ & $62 \pm 5$ & $4980 \pm 1800$ \\
& $\left\langle N_{\mathrm{LHCII}}\right\rangle=4, \mathrm{pH} 7.5$ & $-50 \pm 4$ & $165 \pm 20$ & $50 \pm 4$ & $5800 \pm 2450$ \\
& $\left\langle N_{\mathrm{LHCII}}\right\rangle=4, \mathrm{pH} 5$ & $-49 \pm 5$ & $165 \pm 20$ & $51 \pm 5$ & $4510 \pm 1400$ \\
\hline
\end{tabular}




\section{REPRODUCIBILITY OF THE WAITING TIME TRACES FROM 2D SPECTRA}
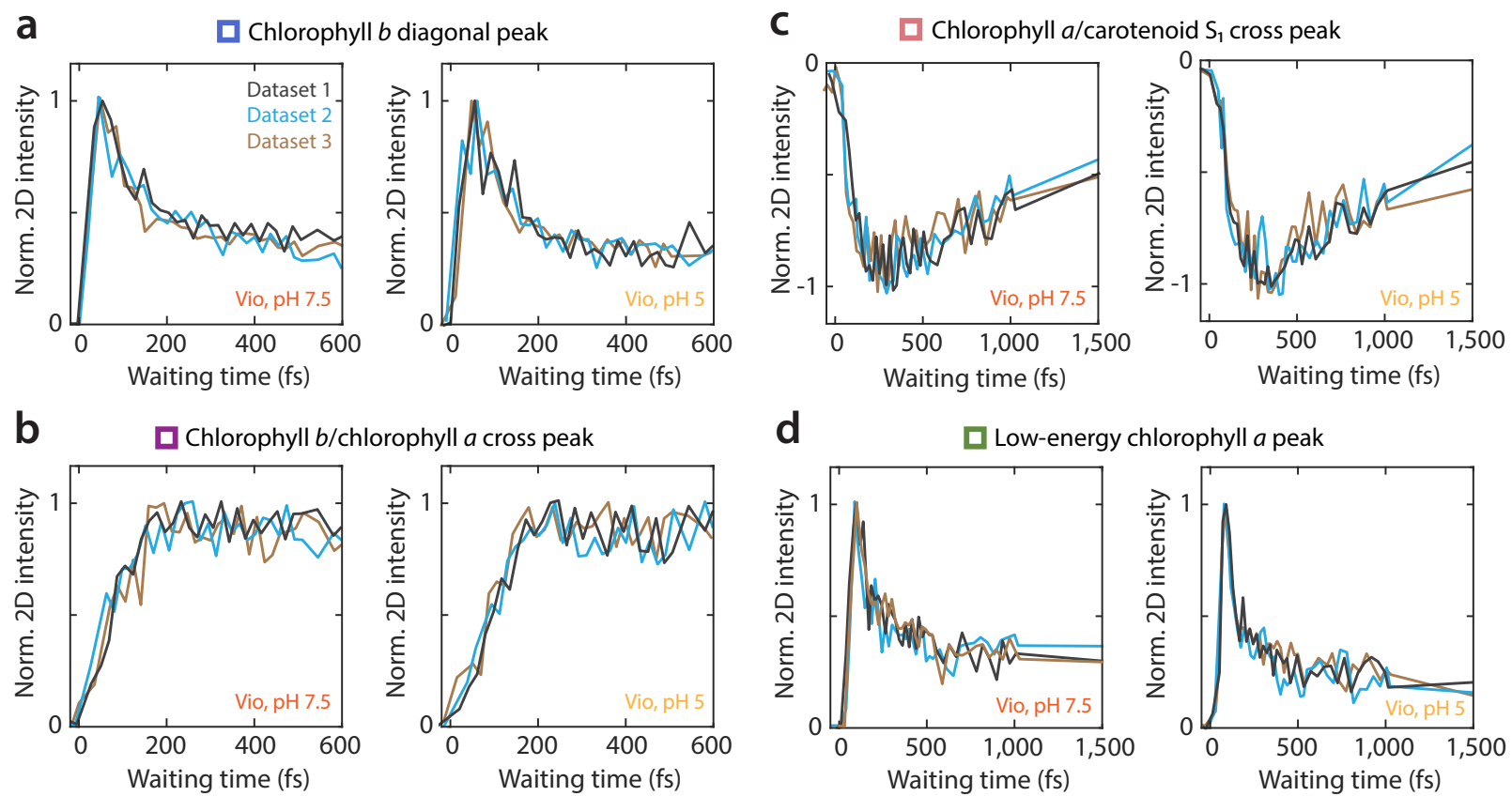

Figure S15: Overlay of waiting time traces from replicate measurements in the chlorophyll region.

(a), (b) and (c), (d) show overlays for the traces shown in Figure 3b and 3c of the main text, respectively, for $\left\langle N_{\mathrm{LHCII}}\right\rangle=4$ nanodiscs. The traces shown in Figure 3b, c are plotted in black (dataset 1). Each trace is normalized to the maximum (minimum) for positive (negative) features, respectively. 

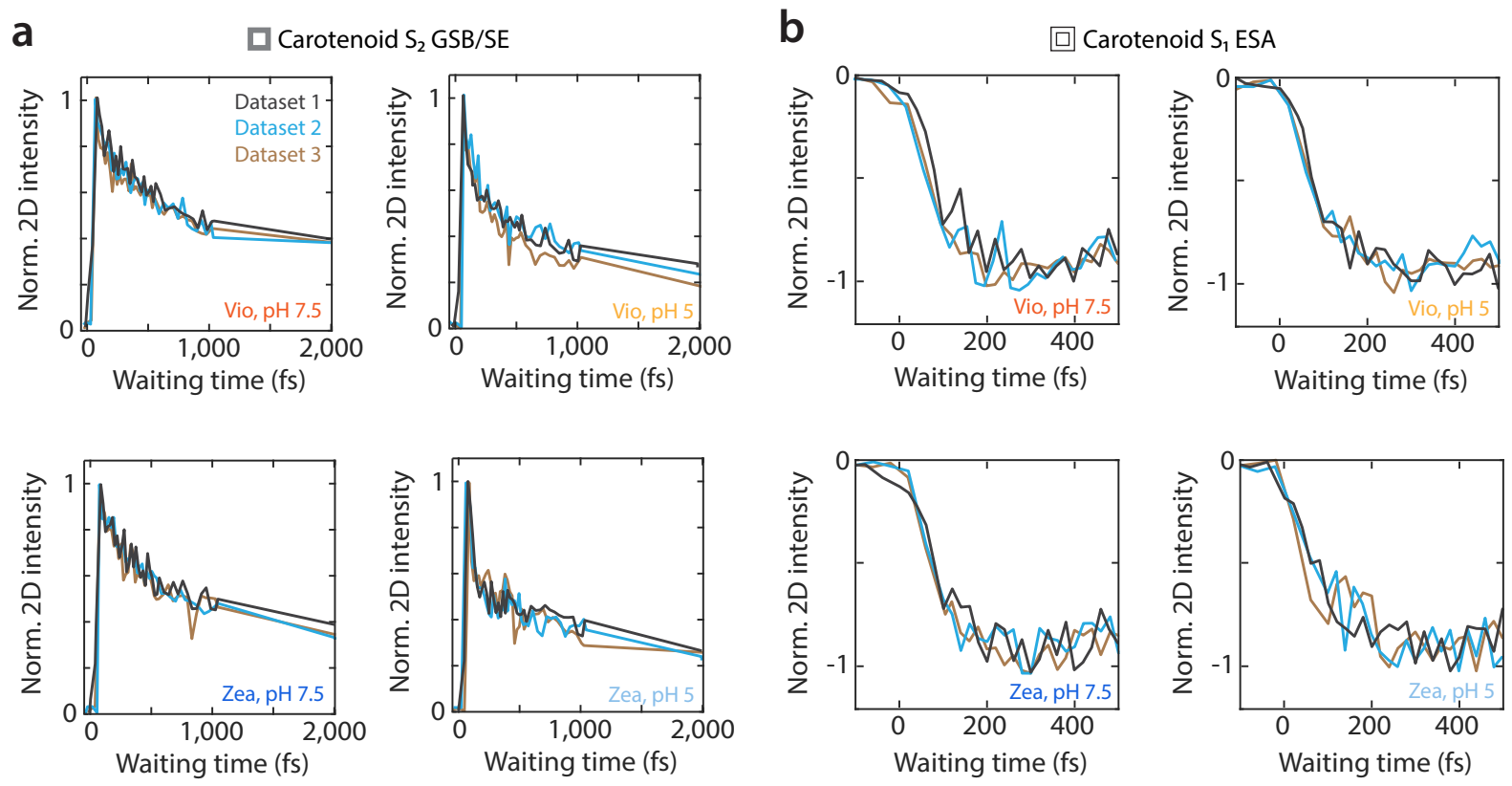

Figure S16: Overlay of waiting time traces from replicate measurements in the carotenoid region.

(a) and (b) show overlays for the traces shown in Figure $4 \mathrm{~b}$ and $4 \mathrm{c}$ of the main text, respectively, for $\left\langle N_{\mathrm{LHCII}}\right\rangle=4$ nanodiscs. The traces shown in Figure $4 \mathrm{~b}, \mathrm{c}$ are plotted in black (dataset 1 ). Each trace is normalized to the maximum (minimum) for positive (negative) features, respectively. 


\section{SUPPLEMENTARY REFERENCES}

1. Bassi, R.; Simpson, D. Chlorophyll-Protein Complexes of Barley Photosystem I. FEBS J. 1987, 163, 221-230.

2. Gilmore, A. M.; Yamamoto, H. Y. Zeaxanthin Formation and Energy-Dependent Fluorescence Quenching in Pea Chloroplasts under Artificially Mediated Linear and Cyclic Electron Transport. Plant Physiol. 1991, 96, 635-643.

3. Morrow, J. A.; Arnold, K. S.; Weisgraber, K. H. Functional Characterization of Apolipoprotein E Isoforms Overexpressed in Escherichia coli. Protein Expr. Purif. 1999, 16, 224-230.

4. Pandit, A.; Shirzad-Wasei, N.; Wlodarczyk, L. M.; van Roon, H.; Boekema, E. J.; Dekker, J. P.; de Grip, W. J. Assembly of the Major Light-Harvesting Complex II in Lipid Nanodiscs. Biophys. J. 2011, 101, 2507-2515.

5. Crisafi, E.; Pandit, A. Disentangling Protein and Lipid Interactions that Control a Molecular Switch in Photosynthetic Light Harvesting. Biochim Biophys Acta - Biomembr. 2017, 1859, 40-47.

6. Sakurai, I.; Shen, J.-R.; Leng, J.; Ohashi, S.; Kobayashi, M.; Wada, H. Lipids in Oxygen-Evolving Photosystem II Complexes of Cyanobacteria and Higher Plants. J. Biochem. 2006, 140, 201-209.

7. Son, M.; Pinnola, A.; Gordon, S. C.; Bassi, R.; Schlau-Cohen, G. S. Observation of Dissipative Chlorophyll-toCarotenoid Energy Transfer in Light-Harvesting Complex II in Membrane Nanodiscs. Nat. Commun. 2020, 11, 1295.

8. Boekema, E. J. Negative Staining of Integral Membrane Proteins. Micron Microscop. Acta 1991, 22, 361-369.

9. Perri, A.; Gaida, J. H.; Farina, A.; Preda, F.; Viola, D.; Ballottari, M.; Hauer, J.; De Silvestri, S.; D’Andrea, C.; Cerullo, G.; Polli, D. Time-and Frequency-Resolved Fluorescence with a Single TCSPC Detector via a FourierTransform Approach. Opt. Express 2018, 26, 2270-2279.

10. van Oort, B.; Roy, L. M.; Xu, P.; Lu, Y.; Karcher, D.; Bock, R.; Croce, R. Revisiting the Role of Xanthophylls in Nonphotochemical Quenching. J. Phys. Chem. Lett. 2018, 9, 346-352.

11. Mascoli, V.; Liguori, N.; Xu, P.; Roy, L. M.; van Stokkum, I. H. M.; Croce, R. Capturing the Quenching Mechanism of Light-Harvesting Complexes of Plants by Zooming in on the Ensemble. Chem 2019, 5, 2900-2912.

12. Luchowski, R.; Gryczynski, Z.; Sarkar, P.; Borejdo, J.; Szabelski, M.; Kapusta, P.; Gryczynski, I. Instrument Response Standard in Time-Resolved Fluorescence. Rev. Sci. Instrum. 2009, 80, 033109.

13. Schwille, P.; Haupts, U.; Maiti, S.; Webb, W. W. Molecular Dynamics in Living Cells Observed by Fluorescence Correlation Spectroscopy with One-and Two-Photon Excitation. Biophys. J. 1999, 77, 2251-2265.

14. Son, M.; Mosquera-Vázquez, S.; Schlau-Cohen, G. S. Ultrabroadband 2D Electronic Spectroscopy with HighSpeed, Shot-to-Shot Detection. Opt. Express 2017, 25, 18950-18962.

15. Trebino, R.; DeLong, K. W.; Fittinghoff, D. N.; Sweetser, J. N.; Krumbügel, M. A.; Richman, B. A.; Kane, D. J. Measuring Ultrashort Laser Pulses in the Time-Frequency Domain Using Frequency-Resolved Optical Gating. Rev. Sci. Instrum. 1997, 68, 3277-3295.

16. Duan, H.-G.; Stevens, A. L.; Nalbach, P.; Thorwart, M.; Prokhorenko, V. I.; Miller, R. J. D. Two-Dimensional Electronic Spectroscopy of Light-Harvesting Complex II at Ambient Temperature: A Joint Experimental and Theoretical Study. J. Phys. Chem. B 2015, 119, 12017-12027. 